\title{
Assessment of the PETase Conformational Changes Induced by Poly(ethylene terephthalate) Binding
}

\author{
Clauber Henrique Costa ${ }^{1}$, Alberto dos Santos ${ }^{2}$, Cláudio Nahum Alves ${ }^{1}$, Sérgio Martí3 \\ Vicente Moliner ${ }^{3}$, Kaue Santana ${ }^{4}$, and Jeronimo Lameira Silva ${ }^{1}$ \\ ${ }^{1}$ Universidade Federal do Pará \\ ${ }^{2}$ Universidade Federal do Maranhão \\ ${ }^{3}$ Universitat Jaume I \\ ${ }^{4}$ Universidade Federal do Oeste do Pará
}

March 9, 2021

\begin{abstract}
Recently, a bacterium strain of Ideonella sakaiensis was identified with the uncommon ability to degrade the poly(ethylene terephthalate) (PET). The PETase from I. sakaiensis strain 201-F6 catalyzes the hydrolysis of PET converting it to mono(2hydroxyethyl) terephthalic acid (MHET), bis(2-hydroxyethyl)-TPA (BHET), and terephthalic acid (TPA). Despite the potential of this enzyme for mitigation or elimination of environmental contaminants, one of the limitations of the use of PETase for PET degradation is the fact that it acts only at moderate temperature due to its low thermal stability. Besides, molecular details of the main interaction of PET in the active site of PETase remains unclear. Herein, molecular docking and molecular dynamics (MD) simulations were applied to analyze structural changes of PETase induced by PET binding. Results from the essential dynamics revealed that $\beta 1-\beta 2$ connecting loop is very flexible. This Loop is located far from the active site of PETase and we suggest that it can be considered for mutagenesis in order to increase the thermal stability of PETase. The free energy landscape (FEL) demonstrates that the main change in the transition between the unbounded to the bounded state is associated with $\beta 7-\alpha 5$ connecting loop, where the catalytic residue Asp206 is located. Overall, the present study provides insights into the molecular binding mechanism of PET into the PETase structure and a computational strategy for mapping flexible regions of this enzyme, which can be useful for the engineering of more efficient enzymes for recycling the plastic polymers using biological systems.
\end{abstract}

\section{Hosted file}

PETase_main_02_03_21.pdf available at https://authorea.com/users/400456/articles/512790assessment-of-the-petase-conformational-changes-induced-by-poly-ethylene-terephthalatebinding 